Nicole Pristera, MD

Department of Cardiovascular Medicine,

Heart, Vascular, and Thoracic Institute,

Cleveland Clinic, Cleveland, $\mathrm{OH}$
Pulkit Chaudhury, MD

Department of Cardiovascular Medicine,

Heart, Vascular, and Thoracic Institute,

Cleveland Clinic, Cleveland, $\mathrm{OH}$
Erik H. Van Iterson, PhD

Department of Cardiovascular Medicine, Heart, Vascular, and Thoracic Institute, Cleveland Clinic, Cleveland, $\mathrm{OH}$
Leslie S. Cho, MD

Director, Women's Cardiovascular Center, Heart, Vascular, and Thoracic Institute, Cleveland Clinic; Professor of Medicine, Lerner College of Medicine of Case Western Reserve University, Cleveland, $\mathrm{OH}$

\title{
Spontaneous coronary artery dissection: Principles of management
}

\section{ABSTRACT}

Spontaneous coronary artery dissection (SCAD) is an acute noniatrogenic tear in the coronary arterial wall, leading to disruption of coronary blood flow and myocardial infarction. Previously considered rare, it is now recognized as a common cause of acute coronary syndrome, particularly in young women. Despite growing awareness of this disease, there is a paucity of data on acute and long-term therapy. This review summarizes the existing literature on treatment of SCAD and describes a comprehensive management strategy.

\section{KEY POINTS}

Diagnosing SCAD requires a high index of suspicion for young patients presenting with acute coronary syndrome.

SCAD is primarily managed medically in clinically stable patients.

Revascularization is recommended only for patients at high risk due to left main coronary artery dissection, ongoing ischemia, severely limited flow, hemodynamic instability, or refractory arrhythmia.

Long-term management includes screening for fibromuscular dysplasia and other arteriopathies, monitoring for recurrence, and cardiac rehabilitation.
Spontaneous coronary artery dissection

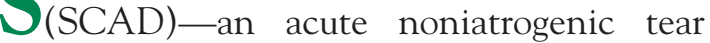
in the coronary artery wall compressing the coronary lumen and possibly causing myocardial infarction - was once thought to be a rare condition but is increasingly recognized as a common cause of acute coronary syndrome (ACS), particularly in young women. ${ }^{1}$

Despite growing awareness of the phenomenon, there is a paucity of data on acute and long-term management. Treatment has traditionally paralleled medical management of atherosclerotic ACS, but the distinct pathophysiology of SCAD behooves us to consider other strategies. A growing body of observational data and retrospective studies is helping to better define SCAD care, but no randomized controlled trials and only a few large-scale prospective studies have focused on management, and few studies have reported outcomes beyond the first few years after dissection. The absence of robust literature has resulted in heterogeneous practice patterns, and guidelines on management remain largely based on expert consensus.

This review summarizes the literature on treatment of SCAD and, based on that, describes a comprehensive management strategy, including the role of revascularization, medical therapy, and long-term follow up.

\section{RISK FACTORS}

The true prevalence of SCAD is unknown, largely due to underdiagnosis. It is overwhelmingly seen in women, particularly young women lacking classic cardiovascular risk factors. Just $10 \%$ of cases occur in men, often following a physical stressor such as exercise or heavy 
lifting. ${ }^{2}$ Studies suggest that SCAD accounts for $1 \%$ to $4 \%$ of cases of ACS overall, ${ }^{3} 15 \%$ to $20 \%$ of peripartum ACS cases, ${ }^{2}$ and up to $35 \%$ of ACS in women under age $60.4,5$

A number of conditions have been associated with SCAD, most notably fibromuscular dysplasia, and less commonly chronic systemic inflammatory disease, connective-tissue disorders, pregnancy, and hypothyroidism. ${ }^{6-8}$ More recently, a few genetic loci have been identified and associated with an increased risk of SCAD. ${ }^{2}$ In addition, various precipitants have been reported including illicit drug use, emotional turmoil, intense exercise, retching, Valsalva maneuver, straining, and other physical stressors. ${ }^{6}$

\section{DIAGNOSIS BY IMAGING}

Diagnosis of SCAD requires a high index of suspicion in all young patients presenting with ACS, particularly women without traditional risk factors for atherosclerosis. A variety of imaging studies are helpful in the diagnosis. However, instrumentation of the vessel wall is associated with risk of propagating the dissection, and intracoronary imaging is thus reserved for clarifying the diagnosis or for guidance during percutaneous coronary intervention $(\mathrm{PCI}) .^{9}$

Revascularization is associated with high failure rates and poor outcomes

\section{Coronary angiography}

As with ACS caused by disruption of atherosclerotic plaque, SCAD is most commonly diagnosed during coronary angiography. However, unlike in plaque rupture, the angiographic appearance of a dissected coronary artery includes multiple radiolucent lines, contrast staining with delayed clearance, and diffuse long narrowing without evidence of significant atherosclerosis apparent in other coronary arteries. ${ }^{10}$ Coronary angiography is regarded as the gold standard to confirm the presence of SCAD, but if not definitive, an adjunctive technique such as intravascular ultrasonography ${ }^{11}$ or optical coherence tomography may be used, with the choice usually based on availability and local expertise.

Optical coherence tomography is particularly useful as it has high spatial resolution $(<10$ $\mu \mathrm{m})$, facilitating detection of true and false lumens, intramural hematoma, and entry tears. ${ }^{12}$ However, it requires additional strong contrast injections and faster pullback speeds that could theoretically extend the dissection plane.

Intravascular ultrasonography is more familiar and more widely available than optical coherence tomography.

Coronary computed tomography angiography is an attractive option due to its ability to demonstrate dissection flaps and intramural hematomas. However, false-negative results are common. ${ }^{13}$ Coronary arterial defects may be subtle, and the distal vessels (often affected by SCAD) are poorly visualized. Current technology has a potential role in evaluating recurrent symptoms or in follow-up, but it has not yet demonstrated adequate sensitivity to rule out SCAD in the acute setting. ${ }^{14}$

\section{MANAGEMENT OF SCAD VS ATHEROSCLEROTIC ACS}

All patients presenting with ACS should be managed in accordance with evidence-based guidelines. Once the diagnosis of SCAD is established, the treatment strategy diverges from that for atherosclerotic ACS. Although culprit-lesion revascularization is the cornerstone of atherosclerotic ACS management, SCAD is primarily managed medically in most clinically stable patients. There are two reasons for this strategy:

- Dissected vessels tend to heal over time: The natural history of SCAD appears to be spontaneous gradual healing of the vessel wall, with complete angiographic resolution of the lesion reported in most cases ( $73 \%$ to $97 \%$ ) within 4 to 6 weeks ${ }^{4,15}$

- Revascularization is associated with high failure rates and poor outcomes in the setting of disrupted arterial wall integrity. Instrumentation of the damaged and friable vessel may worsen the disease and impair the healing process. Passage of the guidewire into a dissected artery risks entry into the false lumen, leading to propagation of dissection ${ }^{15}$ and requiring more stents than expected. ${ }^{16}$ Furthermore, balloon dilation can lead to proximal or distal extension or migration of intramural hematoma, worsening the luminal diameter. Lastly, the eventual healing of the vessel and changing architecture can result in stent malposition and future complications of stent thrombosis over the long term. 


\section{Data supports a conservative approach}

Observational studies have consistently demonstrated that PCI in the setting of SCAD is associated with worse outcomes and high complication rates. ${ }^{17}$

In a retrospective study of 189 patients with SCAD, the procedural failure rate was $53 \%$ in those managed with PCI. ${ }^{18}$ Reasons for technical failure included wire entry into a false lumen, final loss of flow after stent placement, and significant residual stenosis. In addition to procedural failure, PCI was associated with a high risk for emergency coronary artery bypass grafting (CABG), ie, $13 \%$ vs $2 \%$ in the medically managed group. These data are consistent with other observational studies citing high PCI failure rates. In a large Vancouver cohort of 327 patients with SCAD, 54 were treated with PCI; only $43.1 \%$ of procedures were deemed successful. ${ }^{4}$ In a smaller study of 134 patients with SCAD in Italy, the procedure success rate was reported at $72.5 \%$, with a trend toward higher incidence of major adverse cardiovascular events in the invasive group, driven by a higher rate of repeat revascularization. ${ }^{19}$

\section{Revascularization appropriate in some cases}

Despite these often poor outcomes, revascularization procedures may be appropriate in patients with the following high-risk features:

- Left main coronary artery dissection

- Ongoing ischemia

- Thrombolysis in Myocardial Infarction (TIMI) grade $0-1$ flow in a proximal vessel

- Hemodynamic instability

- Refractory arrhythmia. ${ }^{10,20,21}$

The goals of PCI are somewhat different for SCAD than for traditional ACS. The goal of PCI in atherosclerotic ACS is to restore flow to TIMI grade 3, with residual stenosis of $20 \%$ or less. The goal in SCAD is to improve baseline TIMI flow by at least 1 grade or to maintain or achieve TIMI grade 2 or 3 , and to reduce residual stenosis to less than 50\%. ${ }^{22}$

However, neither PCI with intracoronary stenting nor $\mathrm{CABG}$ appears to be protective against recurrent dissection. ${ }^{18,19} \mathrm{~A}$ study that included 20 patients who underwent CABG for SCAD found that at 5 years, 1 patient had recurrent SCAD, 3 had heart failure, and 6 had target-vessel revascularization, presum-

\section{TABLE 1}

\section{Medications for spontaneous coronary artery dissection}

\section{Indicated}

Aspirin $81 \mathrm{mg}$ daily

P2Y12 inhibitor if patient has undergone percutaneous coronary intervention ( $\mathrm{PCI}$ )

Beta-blocker

Debated

P2Y12 inhibitor if no PCl: consider a 1- to 3-month course as tolerated

Statin (appropriate if otherwise indicated)

Angiotensin-converting enzyme inhibitor or angiotensin receptor blocker in patients with left ventricular dysfunction

\section{Contraindicated}

Thrombolysis

ably secondary to healing of native coronary arteries resulting in competitive flow. ${ }^{18}$

\section{Mechanical support for shock}

For SCAD complicated by cardiogenic shock, mechanical circulatory support may be considered in accordance with consensus guidelines for non-SCAD ACS treatment. ${ }^{23}$ While case reports suggest that an intraaortic balloon pump and extracorporeal membrane oxygenation can be used safely in patients with SCAD, ${ }^{24,25}$ they should be used with caution because, given the high incidence of concomitant arteriopathies in patients with SCAD, insertion of large-bore arterial catheters can theoretically result in iatrogenic dissection of the iliac arteries or aorta.

\section{MEDICAL THERAPY}

Medical therapy recommendations are summarized in Table 1.

\section{Antiplatelet therapy}

In the absence of randomized controlled trials to guide antiplatelet therapy for SCAD, data from traditional atherosclerotic ACS literature are extrapolated for this patient population. Aspirin is widely used based on its favorable side-effect profile and extensive literature supporting its benefits in traditional athero- 
sclerotic ACS. ${ }^{26}$ Patients who undergo stent placement should be treated in accordance with current ACS guidelines and receive dual antiplatelet therapy (DAPT) consisting of aspirin and a P2Y12 inhibitor for 12 months.

For patients who do not undergo PCI, the addition of a second antiplatelet agent is controversial. Expert consensus states that a course of DAPT may be considered ${ }^{2}$ with the goal of minimizing thrombus burden and maintaining patency of the true lumen. While clopidogrel is most commonly prescribed, evidence is limited comparing clopidogrel, ticagrelor, and prasugrel in SCAD. Furthermore, the optimal duration of DAPT is unknown. Some authors recommend treatment for up to 12 months, while others advocate discontinuing the P2Y12 inhibitor after 1 to 3 months or when healing of the dissection is confirmed. ${ }^{27}$

Although DAPT in SCAD has been shown to be safe in several observational studies, there is a theoretical concern about the use of antiplatelet agents in a disease state that may be triggered by intramural bleeding. ${ }^{28}$ In a cohort of 64 patients with SCAD, ${ }^{29} 59$ (92\%) received DAPT with aspirin plus either clopidogrel $(69 \%)$, prasugrel $(14 \%)$, or ticagrelor

Thrombolysis is contraindicated in any patient with SCAD as it may propagate dissection and lead to coronary rupture and cardiac tamponade
$(9 \%)$. Of the 40 patients who underwent repeat angiography, healing of dissection was demonstrated in all but 1. DAPT was well tolerated, with no specific medication-related complications noted. ${ }^{29}$

As of this writing, no data have been published on the use of glycoprotein IIb/IIIa inhibitors in SCAD.

\section{Limited role for anticoagulation}

In line with contemporary ACS guidelines, anticoagulation is often appropriately initiated before SCAD is diagnosed. Once SCAD is identified as the cause of ACS, there is no clear benefit to continuing this therapy. ${ }^{2}$ Anticoagulation involves a theoretical risk of increased intramural bleeding and extension of dissection, although there is a paucity of evidence on this. According to expert consensus, anticoagulants should be stopped upon confirmation of SCAD in the absence of another compelling indication for anticoagulation such as left ventricular thrombus or other thromboembolic disease. ${ }^{28}$ The few published case reports on SCAD complicated by left ventricular thrombus report safe treatment with anticoagulation. ${ }^{30}$

\section{No role for thrombolysis}

Thrombolysis is contraindicated in SCAD, as it may propagate dissection and lead to coronary rupture and cardiac tamponade. ${ }^{28}$ Several case reports have been published documenting the adverse effects of thrombolysis in SCAD. ${ }^{31,32}$

\section{Beta-blockers as tolerated}

Beta-blockers are central to the management of acute aortic dissection, reducing shear stress on the vessel wall and minimizing risk of propagation. ${ }^{33}$ It follows that they would be similarly beneficial for SCAD. Beta-blockers serve not only to lower blood pressure but also to modulate heart rate, the cornerstone of impulse control. In a recent study of 327 patients with SCAD, beta-blocker use was associated with reduced risk of recurrent SCAD (hazard ratio $0.36, P=.004) .{ }^{4}$ If validated in future studies, these findings would provide the first evidence for recurrence risk-reduction through medical therapy.

In practice, the use of beta-blockers is often limited by hypotension and fatigue, especially as patients are often young women without coexisting hypertension. ${ }^{10}$ No studies to date have evaluated the efficacy of different types of beta-blockers, goal heart rate, or blood pressure after SCAD. Nonetheless, it is reasonable to escalate beta-blocker therapy to the maximally tolerated dose. In patients unable to tolerate beta-blockers, a nondihydropyridine calcium channel blocker should be considered.

\section{Lipid-lowering therapy if otherwise indicated}

As no proven connection has been identified between cholesterol and risk of SCAD, statins and other lipid-lowering agents are generally reserved for patients with traditional indications for those medications. In a retrospective single-center cohort study of 87 patients, statin use was associated with subsequent risk of SCAD recurrence. ${ }^{17}$ However, as this analysis was limited by small sample size and incomplete information on statin use, it should be interpreted with caution. The signal for increased recurrence of SCAD with statin use has not been demonstrated in larger stud- 
ies. ${ }^{4}$ In the absence of any other indication for lipid-lowering therapy, we do not routinely prescribe statins in patients with SCAD.

\section{LONG-TERM MANAGEMENT}

Chronic management of SCAD is based on several key principles, ie, screening for fibromuscular dysplasia, monitoring for chest pain and recurrence, and cardiac rehabilitation.

\section{Screen for fibromuscular dysplasia and other arteriopathies}

Fibromuscular dysplasia is an idiopathic arteriopathy not caused by underlying atherosclerosis or inflammation, with a predilection for medium-sized vessels. It is the condition most commonly associated with SCAD, with an estimated prevalence of $25 \%$ to $86 \% .^{34}$ This wide range reflects differences between screening methods and the number of vascular territories screened. The hallmark feature on imaging is the "string of beads," which occurs where areas of fibrosis (causing narrowing) alternate with regions of dilation. ${ }^{35}$ The renal, carotid, and vertebral arteries are most often affected, but nearly any site may be involved.

Other arteriopathies associated with SCAD include Marfan syndrome, Loeys-Dietz syndrome, vascular Ehlers-Danlos syndrome, alpha-1 antitrypsin deficiency, and polycystic kidney disease. ${ }^{7,8}$

Given these associations, a vascular medicine evaluation is recommended for all patients diagnosed with SCAD. The evaluation should include a comprehensive vascular history, examination, and brain-to-pelvis imaging. ${ }^{10}$ Patients with a family history or physical examination findings suggestive of known arteriopathies may benefit from a genetics evaluation. Coronary computed tomography angiography is preferred for imaging when possible, as it has higher spatial resolution than magnetic resonance angiography or ultrasonography. Screening provides valuable data to guide management, develop a longitudinal follow-up plan, and inform prognosis.

\section{Monitor for chest pain and recurrence}

Although the prognosis for long-term survival is favorable, patients are at risk of chronic angina, recurrent SCAD, and noncardiac chest pain. Optimal management requires regular follow-up with a cardiologist experienced in the care of SCAD.

Chronic angina. Of the approximately $20 \%$ of patients with SCAD who are readmitted within 30 days of the index event, many develop chronic nitrate-responsive chest pain. ${ }^{3}$ This is suspected to be related to coronary microvascular dysfunction, which is common in this population. In a small study of 17 patients undergoing coronary flow reserve testing on coronary angiography at least 3 months post-SCAD, more than $70 \%$ had coronary microvascular dysfunction defined by a coronary flow reserve $<2.5$ or an index of microcirculatory resistance $>25$ units. ${ }^{36} \mathrm{~A}$ long-acting nitrate or calcium channel blocker, or both, may be considered as needed.

Recurrent SCAD. In a prospective series of 327 patients, the recurrent SCAD rate was $10.4 \%$ over a median follow-up of 3.1 years. ${ }^{4}$ Higher recurrence rates have been reported, with one retrospective study of 189 patients finding 27\%. ${ }^{18}$ Patients should therefore be monitored closely for new or worsening cardiac symptoms, which should prompt further testing.

While no consensus-based recommendations for a particular noninvasive imaging method have been developed, we find positron emission tomography stress imaging with coronary flow reserve most helpful, as it allows evaluation of ischemia in the previously affected territory.

Coronary computed tomography angiography can be beneficial for follow-up, particularly in patients with known large-vessel proximal SCAD and concern for recurrence soon after the index event. The main limitation with this imaging method is the possibility of missing middle or distal small-vessel disease. ${ }^{13}$

Lastly, cardiac magnetic resonance imaging is useful in stable patients with recent SCAD and suspicion for pericarditis. There is growing interest in its use as a surveillance tool, with evidence that it can be used to quantify infarct size. ${ }^{37}$ Further research is needed to define its role in SCAD.

\section{Recommend cardiac rehabilitation}

Cardiac rehabilitation is an important component of management following SCAD, but it remains significantly underused. Young and otherwise healthy women, comprising the ma-

\section{A vascular medicine evaluation is recommended for all patients diagnosed with SCAD}




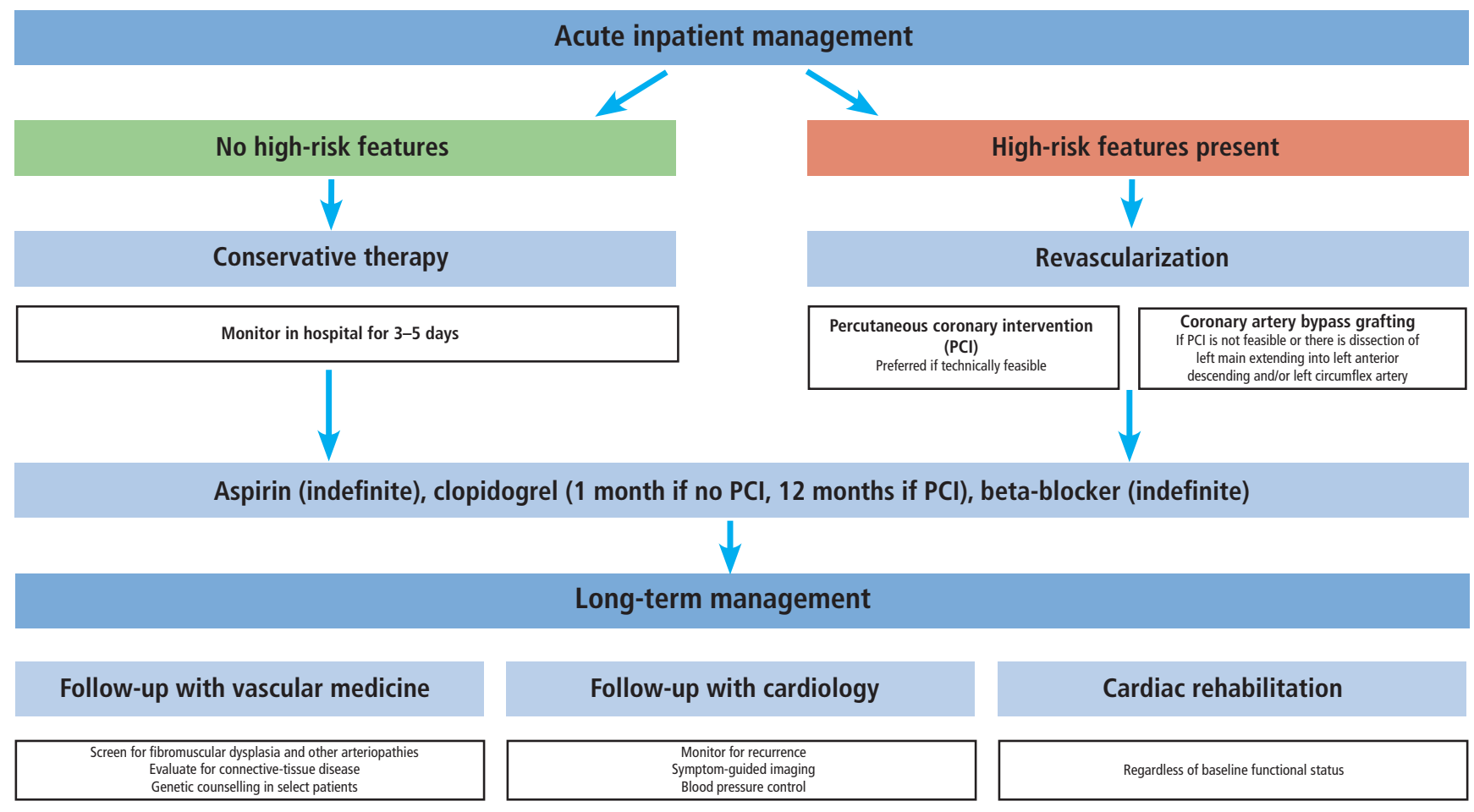

Figure 1. Our approach to the management of spontaneous coronary artery dissection.

Isometric
resistance
training should
be avoided;
strength
training
should be
light-intensity

jority of patients with SCAD, are infrequently referred for cardiac rehabilitation. There is also concern about the safety of cardiac rehabilitation in light of the association of SCAD with physical activity. ${ }^{17}$ Collectively, these barriers result in low enrollment, adherence, and completion rates.

However, cardiac rehabilitation has clear short-term and long-term benefits for perceived physical and mental health, and it has consistently been shown to be safe in patients with SCAD. ${ }^{38,39}$ In a cohort of 70 women with SCAD participating in cardiac rehabilitation, improvements were found in exercise capacity, symptoms (specifically chest pain), and psychosocial well-being at the conclusion of the program..$^{39}$ Similarly, of the 269 patients who participated in cardiac rehabilitation from the Mayo Clinic SCAD registry, $82 \%$ reported physical health benefits and $75 \%$ reported emotional health benefits. ${ }^{40}$

We recommend referring all patients with SCAD for cardiac rehabilitation, including young women without other comorbidities. Our aerobic exercise plan involves training set at a target heart rate zone equivalent to $50 \%$ to $70 \%$ heart rate reserve based on initial exercise stress testing. The frequency of cardiac rehabilitation and aerobic exercise participation should be at least 3 times weekly for 20 to 30 minutes per session as a starting point. As patient fitness and comfort with exercise gradually improve, program progression should be encouraged but implemented conservatively and without including high-intensity interval-training methods. The long-term goal is for patients to comfortably exercise 45 to 60 minutes per session on most days of the week.

Resistance training involving isometric (constant muscle length) contraction, particularly using large muscle groups, should be avoided in patients with a history of SCAD, as these activities are often associated with temporary loading of muscles, closely followed by acute periods of high power and pressure generation.

Similarly, strength training should be limited to light intensity. Greater intensity is typically associated with Valsalva-type maneuvers, which rapidly generate transient bursts of high-to-extreme levels of intrathoracic, 
cardiac, and aortic pressures. This yields high levels of localized vascular wall mechanical shear stress and compensatory spikes in heart rate, all of which should be avoided in patients with a history of SCAD.

\section{OUR APPROACH}

At our institution, we use a stepwise approach to the treatment of SCAD (Figure 1) that starts with assessing the patient for high-risk features that may prompt an invasive strategy for management rather than medical management. Then medical therapy is started as outlined in Table 1. All patients with SCAD should undergo a vascular medicine evaluation and should be followed regularly by a cardiologist with expertise in SCAD. In addition, referral for cardiac rehabilitation is essential, offering physical and mental health benefits.

\section{NEEDED: MORE EVIDENCE FOR BEST MANAGEMENT}

Prospective and randomized-controlled studies are needed to facilitate development of an evidence-based treatment algorithm for SCAD. In particular, studies to investigate the role and duration of DAPT, use of statin therapy, and indications for and timing of revascularization would greatly enhance management. With increasing clinician awareness of SCAD, coupled with advancement in angiographic diagnostic techniques, the prevalence of this disease will likely continue to grow. Hence, the need for a clear treatment approach becomes all the more pressing.

\section{DISCLOSURES}

Dr. Cho has disclosed consulting and research/independent contracting for Amgen, AstraZeneca Pharmaceuticals, and Esperion, and research/independent contracting for Novartis Pharmaceuticals. The other authors report no financial relationships which, in the context of their contributions, could be perceived as a potential conflict of interest.

\section{REFERENCES}

1. Lebrun S, Bond RM. Spontaneous coronary artery dissection (SCAD) the underdiagnosed cardiac condition that plagues women. Trends Cardiovasc Med 2018; 28(5):340-345. doi:10.1016/j.tcm.2017.12.004

2. Kim ESH. Spontaneous coronary-artery dissection. N Engl J Med 2020; 383(24):2358-2370. doi:10.1056/NEJMra2001524

3. Gad MM, Mahmoud AN, Saad AM, et al. Incidence, clinical presentation, and causes of 30-day readmission following hospitalization with spontaneous coronary artery dissection. JACC Cardiovasc Interv 2020; 13(8):921-932. doi:10.1016/j.jcin.2019.12.033

4. Saw J, Humphries K, Aymong E, et al. Spontaneous coronary artery dissection: clinical outcomes and risk of recurrence. J Am Coll Cardiol 2017; 70(9):1148-1158. doi:10.1016/j.jacc.2017.06.053

5. Gulati R, Behfar A, Narula J, et al. Acute myocardial infarction in young individuals. Mayo Clin Proc 2020; 95(1):136-156. doi:10.1016/j.mayocp.2019.05.001

6. Hayes SN, Tweet MS, Adlam D, et al. Spontaneous coronary artery dissection: JACC state-of-the-art review. J Am Coll Cardiol 2020; 76(8):961-984. doi:10.1016/j.jacc.2020.05.084

7. Motreff P, Malcles G, Combaret N, et al. How and when to suspect spontaneous coronary artery dissection: novel insights from a singlecentre series on prevalence and angiographic appearance. EuroIntervention 2017; 12(18):e2236-e2243. doi:10.4244/EIJ-D-16-00187

8. Nakashima T, Noguchi T, Haruta $S$, et al. Prognostic impact of spontaneous coronary artery dissection in young female patients with acute myocardial infarction: a report from the Angina Pectoris-Myocardial Infarction Multicenter Investigators in Japan. Int J Cardiol 2016; 207:341-348. doi:10.1016/j.ijcard.2016.01.188

9. Macaya F, Salazar CH, Pérez-Vizcayno MJ, et al. Feasibility and safety of intracoronary imaging for diagnosing spontaneous coronary artery dissection. JACC Cardiovasc Imaging 2019; 12(4):763-764. doi:10.1016/j.jcmg.2018.09.023

10. Hayes SN, Kim ESH, Saw J, et al. Spontaneous coronary artery dissection: current state of the science: a scientific statement from the American Heart Association. Circulation 2018; 137(19):e523-e557. doi:10.1161/CIR.0000000000000564

11. Arnold JR, West NE, van Gaal WJ, Karamitsos TD, Banning AP. The role of intravascular ultrasound in the management of spontaneous coronary artery dissection. Cardiovasc Ultrasound 2008; 6:24. doi:10.1186/1476-7120-6-24

12. Alfonso F, Paulo M, Gonzalo N, et al. Diagnosis of spontaneous coronary artery dissection by optical coherence tomography. J Am Coll Cardiol 2012; 59(12):1073-1079. doi:10.1016/j.jacc.2011.08.082

13. Eleid MF, Tweet MS, Young PM, Williamson E, Hayes SN, Gulati R. Spontaneous coronary artery dissection: challenges of coronary computed tomography angiography. Eur Heart J Acute Cardiovasc Care 2018; 7(7):609-613. doi:10.1177/2048872616687098

14. Gilhofer TS, Saw J. Spontaneous coronary artery dissection: update 2019. Curr Opin Cardiol 2019; 34(6):594-602. doi:10.1097/HCO.0000000000000671

15. Main A, Saw J. Percutaneous coronary intervention for the treatment of spontaneous coronary artery dissection. Interv Cardiol Clin 2019; 8(2):199-208. doi:10.1016/j.iccl.2018.11.008

16. Tweet MS, Kok SN, Hayes SN. Spontaneous coronary artery dissection in women: what is known and what is yet to be understood. Clin Cardiol 2018; 41(2):203-210. doi:10.1002/clc.22909

17. Tweet MS, Hayes SN, Pitta SR, et al. Clinical features, management, and prognosis of spontaneous coronary artery dissection. Circulation 2012; 126(5):579-588. doi:10.1161/CIRCULATIONAHA.112.105718

18. Tweet MS, Eleid MF, Best PJ, et al. Spontaneous coronary artery dissection: revascularization versus conservative therapy. Circ Cardiovasc Interv 2014; 7(6):777-786. doi:10.1161/CIRCINTERVENTIONS.114.001659

19. Lettieri C, Zavalloni D, Rossini R, et al. Management and long-term prognosis of spontaneous coronary artery dissection. Am J Cardiol 2015; 116(1):66-73. doi:10.1016/j.amjcard.2015.03.039

20. Graham RM, McGrath-Cadell L, Muller DWM, Holloway CJ. The mystery and enigma of spontaneous coronary artery dissection. Heart Lung Circ 2018; 27(4):401-405. doi:10.1016/S1443-9506(18)30060-X

21. Zingarelli A. Spontaneous coronary dissection: unravelling the complex riddle of conservative or interventional treatment. Int J Cardiol 2017; 229:124. doi:10.1016/j.ijcard.2016.10.065

22. Lobo AS, Cantu SM, Sharkey SW, et al. Revascularization in patients with spontaneous coronary artery dissection and ST-segment elevation myocardial infarction. J Am Coll Cardiol 2019; 74(10):12901300. doi:10.1016/j.jacc.2019.06.065

23. Roffi M, Patrono C, Collet JP, et al. 2015 ESC guidelines for the management of acute coronary syndromes in patients presenting 


\section{SPONTANEOUS CORONARY ARTERY DISSECTION}

without persistent ST-segment elevation. Rev Esp Cardiol (Engl Ed) 2015; 68(12):1125. doi:10.1016/j.rec.2015.10.009

24. Jorge-Pérez P, García-González MJ, Ávalos-Pinto RM, et al. Spontaneous coronary dissection and cardiogenic shock requiring mechanical circulatory support in a non-transplant center. Int J Cardiol 2016; 221:629-630. doi:10.1016/j.ijcard.2016.07.015

25. Weinberg L, Ong M, Tan CO, McDonnell NJ, Lo C, Chiam E. Spontaneous coronary artery dissection in pregnancy requiring emergency caesarean delivery followed by coronary artery bypass grafting. Anaesth Intensive Care 2013; 41(2):251-255. doi:10.1177/0310057X1304100215

26. Amsterdam EA, Wenger NK, Brindis RG, et al. 2014 AHA/ACC guideline for the management of patients with non-ST-elevation acute coronary syndromes: executive summary: a report of the American College of Cardiology/American Heart Association Task Force on Practice Guidelines. Circulation 2014; 130(25):2354-2394. doi:10.1161/CIR.0000000000000133

27. Ahmed Z, Bajwa A, Bhardwaj B, Laster SB, Magalski A. Spontaneous coronary artery dissection: the management dilemma continues. BMJ Case Rep 2015; 2015:bcr2015211061. doi:10.1136/bcr-2015-211061

28. Adlam D, Alfonso F, Maas A, Vrints C; Writing Committee. European Society of Cardiology, acute cardiovascular care association, SCAD study group: a position paper on spontaneous coronary artery dissection. Eur Heart J 2018; 39(36):3353-3368. doi:10.1093/eurheartj/ehy080

29. Rogowski S, Maeder MT, Weilenmann D, et al. Spontaneous coronary artery dissection: angiographic follow-up and long-term clinical outcome in a predominantly medically treated population. Catheter Cardiovasc Interv 2017; 89(1):59-68. doi:10.1002/ccd.26383

30. Joo JH, Caldera AE, Divakaran VG. Spontaneous coronary artery dissection with left ventricular thrombus. Proc (Bayl Univ Med Cent) 2018; 32(1):99-100. doi:10.1080/08998280.2018.1507974

31. Zupan I, Noc M, Trinkaus D, Popovic M. Double vessel extension of spontaneous left main coronary artery dissection in young women treated with thrombolytics. Catheter Cardiovasc Interv 2001;
$52(2) \cdot 226-230$

doi:10.1002/1522-726x(200102)52:2<226::aid-ccd1054>3.0.co;2-r

32. Andreou AY, Georgiou PA, Georgiou GM. Spontaneous coronary artery dissection: Report of two unsuspected cases initially treated with thrombolysis. Exp Clin Cardiol 2009; 14(4):e89-e92. pmid:20198198

33. Mokashi SA, Svensson LG. Guidelines for the management of thoracic aortic disease in 2017. Gen Thorac Cardiovasc Surg 2019; 67(1):59-65. doi:10.1007/s11748-017-0831-8

34. Garcia-Guimarães M, Bastante $T$, Antuña $P$, et al. Spontaneous coronary artery dissection: mechanisms, diagnosis and management. Eur Cardiol 2020; 15:1-8. doi:10.15420/ecr.2019.01

35. Khoury MH, Gornik HL. Fibromuscular dysplasia (FMD). Vasc Med 2017; 22(3):248-252. doi:10.1177/1358863X17700716

36. Sedlak T, Starovoytov A, Humphries K, Saw J. Coronary flow reserve in patients with prior spontaneous coronary artery dissection and recurrent angina. J Am Heart Assoc 2020; 9(16):e015834. doi:10.1161/JAHA.120.015834

37. Al-Hussaini A, Abdelaty AMSEK, Gulsin GS, et al. Chronic infarct size after spontaneous coronary artery dissection: implications for pathophysiology and clinical management. Eur Heart J 2020; 41(23):2197-2205. doi:10.1093/eurheartj/ehz895

38. Silber TC, Tweet MS, Bowman MJ, Hayes SN, Squires RW. Cardiac rehabilitation after spontaneous coronary artery dissection. J Cardiopulm Rehabil Prev 2015; 35(5):328-333. doi: 10.1097/HCR.0000000000000111

39. Chou AY, Prakash R, Rajala J, et al. The first dedicated cardiac rehabilitation program for patients with spontaneous coronary artery dissection: description and initial results. Can J Cardiol 2016; 32(4):554-560. doi:10.1016/j.cjca.2016.01.009

40. Krittanawong C, Tweet MS, Hayes SE, et al. Usefulness of cardiac rehabilitation after spontaneous coronary artery dissection. Am J Cardiol 2016; 117(10):1604-1609. doi:10.1016/j.amjcard.2016.02.034

Address: Leslie S. Cho, MD, Department of Cardiovascular Medicine, Heart, Vascular, and Thoracic Institute, JB1, Cleveland Clinic, 9500 Euclid

Avenue, Cleveland, $\mathrm{OH} 44195 ;$ chol@ccf.org 